Research, Society and Development, v. 9, n. 10, e6499109147, 2020

(CC BY 4.0) | ISSN 2525-3409 | DOI: http://dx.doi.org/10.33448/rsd-v9i10.9147

\title{
Revisão integrativa sobre a importância da Educação em Saúde no aperfeiçoamento dos profissionais de enfermagem
}

\section{Integrative review on the importance of Health Education in the improvement of nursing professionals}

\section{Revisión integradora sobre la importancia de la Educación para la Salud en la superación de los profesionales de enfermería}

Recebido: 08/10/2020 | Revisado: 09/10/2020 | Aceito: 10/10/2020 | Publicado: 12/10/2020

Murilo de Jesus Porto

ORCID: https://orcid.org/0000-0003-2339-8173

Universidade Paulista, Brasil

E-mail: murilo.porto@hotmail.com

David Jesus de Almeida

ORCID: https://orcid.org/0000-0003-1438-1126

Faculdade Independente do Nordeste, Brasil

E-mail: davidalmeida14@hotmail.com

\section{Resumo}

O processo da educação em saúde tem como foco no sentido de saber ensinar a passar uma determinada informação à alguém que não sabe ou que necessita se atualizar sobre determinado tema voltado a aspectos relativos à saúde. O objetivo geral da pesquisa é analisar o que as pesquisas, dos últimos quatros anos, têm proposto sobre a educação continuada em saúde para enfermeiros. Trata-se de um estudo de revisão integrativa, através de busca eletrônica de artigos indexados no SCIELO, a partir da seguinte palavra-chave: educação continuada em saúde aos profissionais de enfermagem, sendo analisado 10 artigos sobre os devidos temas proposto. Como resultados a educação continuada em saúde no âmbito da enfermagem mostra ao entendimento de que o sujeito deve ter o conhecimento atual atingir um determinado objetivo em seguir sua carreira profissional, tal carreira que incluem meios eficazes de aperfeiçoamento para a realização profissional e ofertar a qualidade integral do atendimento a toda sociedade, sempre avaliando a importância e necessidade do paciente como um todo e também sendo viável para a implementação da assistência de enfermagem, possibilitando diálogos humanos os quais condizem aos temas de saúde aos indivíduos havendo os previstos 
cuidados por meio da qualificação. Dessa forma com os resultados dessa pesquisa espera-se contribuir de maneira clara, com os profissionais enfermeiros em geral à respeito de que a qualidade da assistência está intimamente ligada a uma necessidade constante de participação dos mesmos em programas de educação em saúde.

Palavras-chave: Revisão integrativa; Educação em saúde; Profissionais de enfermagem.

\section{Abstract}

The health education process focuses on knowing how to teach people how to pass on certain information to someone who does not know or who needs to update themselves on a specific topic related to aspects related to health. The general objective of the research is to analyze what research, in the last four years, has proposed about continuing education in health for nurses. This is an integrative review study, through electronic search of articles indexed in SCIELO, based on the following keyword: continuing health education for nursing professionals, with 10 articles on the proposed themes being analyzed. As a result, continuing education in health in the field of nursing shows the understanding that the subject must have the current knowledge to achieve a certain objective in pursuing his professional career, such a career that include effective means of improvement for professional achievement and offer comprehensive quality of care for the whole society, always evaluating the importance and need of the patient as a whole and also being viable for the implementation of nursing care, enabling human dialogues which match the health issues to the individuals, with the care provided through qualification. Thus, with the results of this research, it is expected to make a clear contribution, with professional nurses in general, that the quality of care is closely linked to their constant need for participation in health education programs.

Keywords: Integrative review; Health education; Nursing professionals.

\section{Resumen}

El proceso de educación en salud se centra en saber cómo enseñar a las personas cómo transmitir cierta información a alguien que no conoce o que necesita actualizarse sobre un tema específico relacionado con aspectos relacionados con la salud. El objetivo general de la investigación es analizar qué investigaciones, en los últimos cuatro años, han propuesto sobre la educación continua en salud para enfermeras. Se trata de un estudio de revisión integradora, mediante búsqueda electrónica de artículos indexados en SCIELO, con base en la siguiente palabra clave: educación continua en salud para profesionales de enfermería, con 10 artículos sobre los temas propuestos en análisis. Como resultado, la educación continua en salud en el 
campo de la enfermería muestra el entendimiento de que el sujeto debe contar con los conocimientos actuales para lograr un determinado objetivo en el ejercicio de su carrera profesional, una carrera que incluya medios efectivos de superación para el logro profesional y que ofrezca una calidad integral. del cuidado para toda la sociedad, evaluando siempre la importancia y necesidad del paciente en su conjunto y siendo también viable para la implementación del cuidado de enfermería, posibilitando diálogos humanos acordes con la problemática de salud de las personas, con la prestación del cuidado a través de la calificación. Así, con los resultados de esta investigación, se espera hacer un claro aporte, con los profesionales de enfermería en general, que la calidad de la atención está íntimamente ligada a su constante necesidad de participación en programas de educación para la salud.

Palabras clave: Revisión integrativa; Educación para la salud; Profesionales de enfermería.

\section{Introdução}

O aspecto da educação continuada em saúde está voltado aos profissionais que trabalham na rede de saúde em rede geral, tendo o intuito de melhor se profissionalizar através de discussões em atualidades. Dessa maneira, a educação em saúde surgiu para a necessidade e objetivos dos profissionais de saúde em atender a população de forma integral, analisando a necessidade de superar e melhorar o processo do atendimento assistencial, avaliando as precisões de formação e incremento para o trabalho em saúde (Fernandes et al., 2017; Silva et al., 2016 \& Leonor, 2015).

Os progressos técnico-científicos e pedagógicos lidado todos os dias na área da saúde, ao lado das informações contraídas pelo usuário, exige melhoria da qualidade assistencial ganhada. Esta deve ser gerenciada através de atos técnicas e éticas dos profissionais atuantes na saúde em especial a equipe de enfermagem, pendendo, daquilo que aparenta a qualidade dos serviços prestados referentes aos cuidados aos seres humanos, faz-se necessário o cuidado intenso com qualidade. Mas para que isso ocorra é necessário uma qualificação através do conhecimento cientifico (Leonor, 2015 \& Seva-Llo, 2015).

A educação continuada em saúde para enfermeiros analisa o intuito de conscientizar a população em termos de saúde para amenizar os riscos de doenças e óbitos. A educação em saúde está, ligada em uma perspectiva de educação enquanto possibilidade de construir espaços coletivos para análise das ações produzidas durante o processo de trabalho (Ortega et al., 2015; Figueiras, 2014 \& Lavich, 2017). 
O profissional enfermeiro dispõe de métodos de trabalho com aporte científico, através da prestação de cuidados intensos de enfermagem, a qual servirá para articular as demais práticas assistenciais, assim, faz necessário um conhecimento amplo e atualizado sobre as práticas de saúde no intuito de ofertar a qualidade do atendimento em amenizar os riscos de processos patológicos e até mesmo de óbito (Menin, Pettenon, 2015; Leonor, 2015 \& SevaLlo, 2015).

A Educação Continuada em saúde analisa a experiência da atividade profissional relacionado a valorização da prática do saber voltados a didática de saúde em ofertar uma qualidade integral de saúde, vivenciando as necessidades e problemas institucionais, realizando a junção de grupos disponíveis para o diálogo de estímulos de experiências voltado para a didática do saber, e para a melhoria do atendimento (Sardinha-Peixoto et al., 2013).

A interação do enfermeiro em geral requer uma atenção por envolver especificidades e articulações indispensáveis na atualização do cuidado a pacientes distintas necessidades da mais tranquila a mais complexas na qual necessita o tão importante conhecimento científico, manejo tecnológico e humanização (Silva et al., 2016; Menin, Pettenon, 2015 \& Figueiras, Puccini, Silva, 2014).

Contudo, os profissionais atuantes na rede da saúde daquela localidade são os responsáveis pelo processo da prática educativa. Conscientizar profissionais não é uma tarefa fácil, o profissional tem que ter uma habilidade e uma capacidade adequada no aspecto da instrução. A educação continuada em saúde para enfermeiros diz que os profissionais que realizam essa prática, tem que receber uma formação para atuação, neste caso, na realidade isso não é colocado em prática, o que dificulta ainda a orientação e segurança da população quanto ao aprendizado daquele devido tema. Desta forma, ao abreviado da problemática exposta tem-se como questão norteadora: O que os estudos, dos últimos quatro anos, têm descrito sobre a educação continuada em saúde para enfermeiros. Assim, detém ao responder a problemática através do objetivo: analisar o que as pesquisas, dos últimos quatros anos, têm proposto sobre a educação continuada em saúde para enfermeiros.

A pesquisa é relevante por atingir a sociedade como um todo, principalmente aos acadêmicos de enfermagem e profissionais que lidam com a educação continuada em saúde na enfermagem, no intuito de aprimoramento profissional e, consequentemente, a melhoria da qualidade na assistência. É importante ressaltar que a presente pesquisa servirá para agregar o acervo em periódicos em relação ao tema e despertar a realidade moldando a mentalidade dos discentes em formação e profissionais atuantes sobre a educação continuada em saúde. 


\section{Metodologia}

Trata-se de uma revisão integrativa, que foi realizada no mês de julho do ano de 2020. Desta forma, foi analisado os presentes dados dos periódicos, agrupando o leque de propósitos: conceito sobre a temática discutida, análise de referenciais teóricos, e avaliação de problemas metodológicos.

A pesquisa qualitativa é a possibilidade de averiguar e abranger por meio de dados estatísticos de determinados fenômenos retornados para a inteligência, a intuição e a subjetividade, tendo como análise primordial a exposição das características de uma determinada população e também o aperfeiçoamento de ideias (Minayo, 2010).

A classificação da pesquisa bibliográfica ocorreu na Biblioteca Virtual em Saúde na base de dados caracterizada como na Área de Enfermagem do Brasil referindo na rede SCIELO. Desta forma, para a inclusão dos artigos, foram analisados os Descritores em Saúde: educação continuada em saúde para enfermeiros. Os presentes descritores escolhidos foram ajustados, de acordo com a base de dados.

Como critérios de inclusão: artigos dos últimos 4 anos; publicados no Brasil, periódicos com descritores em educação continuada para enfermeiros. Já os critérios de exclusão: periódicos inferiores ao ano de 2017. Também foram excluídos trabalhos como teses, dissertações, livros e capítulos de livros. Sendo totalizado a leitura de nove artigos para a análise de discussão para atingir o devido objetivo.

Para realização desta revisão integrativa, foram utilizadas as seguintes etapas: verificação da questão temática, estabelecimento dos critérios para a seleção da amostra, análise e interpretação dos resultados e apresentação da revisão. Os procedimentos adotados para a presente pesquisa percorreu o seguinte caminho: primeiramente foi inserida no SCIELO a seguinte frase: Educação continuada em saúde para enfermeiros, desta pesquisa surgiram 39 artigos, posteriormente foi realizado o filtro referente ao ano, sendo definido estudos que foram publicados entre os anos de 2017 a 2020, sendo que dessa filtragem restaram apenas nove artigos. Ressalta-se que todos os nove artigos foram identificados para a inserção neste presente estudo. Após este percurso, foi realizada a leitura dos artigos e realizado o preenchimento do questionário conforme objetivo definido. 


\section{Resultados e Discussão}

Os estudos selecionados estão em língua portuguesa, totalizando 9 periódicos; foram publicados no período de 2017 a 2020, em revistas brasileiras, algumas de circulação internacional, tais como: rev. latino-am de enfermagem, esc. anna nery, saúde em debate, texto contexto enferm, trabalho, educação e saúde e revista ciência \& saúde coletiva. O Quadro 1 abaixo mostra a distribuição dos periódicos pesquisados, avaliando o título, ano de publicação e principais descritores: 
Quadro 1- Distribuição do perfil dos periódicos encontrados na Scientific Eletronic Library.

\begin{tabular}{|c|c|c|c|}
\hline $\begin{array}{l}\mathrm{N}^{\mathbf{0}} \\
\text { ARTIGO }\end{array}$ & TITULO DO ARTIGO & $\begin{array}{c}\text { ANO DE } \\
\text { PUBLICAÇÃO }\end{array}$ & DESCRITORES \\
\hline 1 & $\begin{array}{c}\text { Ações De Educação Permanente Dos } \\
\text { Enfermeiros Facilitadores De Um } \\
\text { Núcleo De Educação Em } \\
\text { Enfermagem }\end{array}$ & 2017 & $\begin{array}{l}\text {-Educação continuada; } \\
\text {-Enfermagem. }\end{array}$ \\
\hline 2 & $\begin{array}{c}\text { Capacidade De Inferência } \\
\text { Diagnóstica De Enfermeiras } \\
\text { Especialistas }\end{array}$ & 2017 & $\begin{array}{l}\text { - Estudos de avaliação; } \\
\text { - Diagnósticos de } \\
\text { Enfermagem. }\end{array}$ \\
\hline 3 & $\begin{array}{l}\text { Metodologia para articular processos de } \\
\text { formação-intervenção -avaliação na } \\
\text { educação profissional em enfermagem }\end{array}$ & 2020 & $\begin{array}{l}\text {-Capacitação profissional; } \\
\text {-Educação em saúde; } \\
\text {-Enfermagem; } \\
\text {-Trabalho em saúde. }\end{array}$ \\
\hline 4 & $\begin{array}{c}\text { Percepção De Enfermeiros Sobre O } \\
\text { Cuidado Nutricional À Criança Na } \\
\text { Estratégia Saúde Da Família }\end{array}$ & 2020 & $\begin{array}{l}\text { - Atenção Primária à Saúde; } \\
\text {-Estratégia Saúde da Família; } \\
\text {-Serviços de saúde. Programas } \\
\text { e políticas de nutrição e } \\
\text { alimentação. }\end{array}$ \\
\hline 5 & $\begin{array}{l}\text { Competências Dos Enfermeiros Na } \\
\text { Estratégia Saúde Da Família }\end{array}$ & 2020 & $\begin{array}{l}\text {-Competência Profissional; } \\
\text {-Estratégias Locais; } \\
\text {-Enfermeiras de Saúde da } \\
\text { Família; } \\
\text {-Estratégia Saúde da Família. }\end{array}$ \\
\hline 6 & $\begin{array}{l}\text { Processo Educativo Com Familiares De } \\
\text { Crianças Com Necessidades Especiais } \\
\text { De Saúde Na Transição Hospital-Casa }\end{array}$ & 2020 & $\begin{array}{l}\text { - Enfermagem pediátrica. } \\
\text { Cuidadores; } \\
\text {-Educação em saúde. Doença } \\
\text { crônica; } \\
\text {-Crianças com deficiência. }\end{array}$ \\
\hline 7 & $\begin{array}{l}\text { Análise Da Produção Tecnológica De } \\
\text { Três Programas De Mestrado } \\
\text { Profissional Na Área Da Enfermagem }\end{array}$ & 2020 & $\begin{array}{l}\text { - Educação de Pós-Graduação } \\
\text { em Enfermagem; } \\
\text {-Educação Profissionalizante; } \\
\text {-Enfermagem; } \\
\text {-Pesquisa Aplicada. }\end{array}$ \\
\hline 8 & $\begin{array}{c}\text { Educação Permanente Em Saúde: } \\
\text { Práticas Desenvolvidas Nos Municípios } \\
\text { Do Estado De Goiás }\end{array}$ & 2020 & $\begin{array}{l}\text { - Educação continuada; } \\
\text {-Educação profissional em } \\
\text { saúde pública; } \\
\text {-Saúde pública. }\end{array}$ \\
\hline 9 & $\begin{array}{l}\text { Educação Em Saúde: A Atuação Da } \\
\text { Enfermagem No Ambiente Escolar }\end{array}$ & 2020 & $\begin{array}{l}\text { - Saúde do Adolescente; } \\
\text {-Promoção da Saúde Escolar; } \\
\text {-Educação em Saúde; Serviços } \\
\text { de Saúde Escolar. }\end{array}$ \\
\hline 10 & $\begin{array}{l}\text { Competências Necessárias Ao Gestor De } \\
\text { Unidade De Saúde Da Família: Um } \\
\text { Recorte Da Prática Do Enfermeiro }\end{array}$ & 2020 & $\begin{array}{l}\text { - Atenção Primária à Saúde; } \\
\text {-Estratégia Saúde da Família; } \\
\text {-Competência profissional. }\end{array}$ \\
\hline
\end{tabular}

Fonte: Autoria própria (2020).

Os descritores mais utilizados pelos autores dos artigos relatam a Educação continuada em saúde para enfermeiros juntamente com a responsabilidade do enfermeiro nas unidades de saúde. Ao se verificar os debuxos de pesquisa mais relevantes no padrão pesquisado, identificou-se que alguns utilizaram a abordagem metodológica qualitativa, quantitativa e até mesmo bibliográfica. 
Assim, do quadro acima, constituem de 10 artigos com tema referentes a educação continuada em saúde no âmbito da enfermagem entre os anos de 2017 a 2020, visto que a maioria estão voltados para a rede hospitalar, cuidados assistenciais e práticas educacionais em saúde com descritos com foco em: Enfermagem, Educação em saúde, Assistência e Competência profissional.

Desta forma a amostragem da revisão foi edificada por artigos científicos, selecionados pelos critérios de inclusão previamente estabelecidos, de acordo com as discussões a seguir:

A educação continuada em saúde, mostra a probabilidade das obrigações de desenvolvimento dos profissionais atuantes na área da saúde e a edificação de táticas e processos que melhorem a atenção integral, mantendo forte o domínio social com o desígnio de lançar um impulso positivo a propósito de atender a toda população em termos de saúde tanto individual quanto coletiva

Assim, a tática de criação da educação continuada em saúde, foi iniciada pela gestão municipal, pelo meio do desenvolvimento articular de conhecimentos referentes à saúde integral, para ofertar todo público no intuito de reduzir os índices de morbimortalidade em saúde.

Diante o processo da educação em saúde aos enfermeiros há uma articulação estratégica que proporciona a contenção do processo educativo no habitual da equipe de enfermagem (Precce \& Maraes, 2020). Essas estratégias podem ser avaliadas como um conjunto de ações, decisões que podem ser alcançadas a partir de uma relação do diálogo entre os profissionais que compõem a equipe, essas estratégias visam sempre a qualidade da assistência através dos meios internos de atualizações e mudanças de protocolos em saúde.

Educação em saúde na enfermagem, é viável para a implementação dos diagnósticos de enfermagem através da sistematização da assistência em enfermagem possibilitando que os diálogos humanos condizem aos temas de saúde dos indivíduos havendo os previstos cuidados integrativos da enfermagem por meio da qualificação a atingir decorrências positivas de saúde (Ferreira, Tavares, 2020 \& Barcellos et al., 2020).

Desta maneira é importante enfatizar que os conteúdos abordados têm a função de serem transmitidos pelos profissionais de saúde havendo um domínio do conteúdo abordado, sempre analisando os valores de conhecimentos prévios do público alvo.

No processo ensino aprendizagem a pedagogia é essencial principalmente em considerar a capacidade potencial e dificuldades das construções de sentidos, surgindo novos caminhos para mudanças de acordo com que é discutido e modificando a realidade social naquele âmbito. É importante ressaltar que todos os profissionais necessitam de orientações 
gerais tanto por diálogo quanto por forma impressa, que são de grande ajuda, pois este expediente auxilia no processo de orientação e esclarecimento da comunidade em geral, permitindo reforçar e garantir acesso fácil às orientações fornecidas durante a assistência de enfermagem, tendo em vista que a paciente leva consigo o informativo para tirar eventuais dúvidas no âmbito familiar.

Isso é válido também para os profissionais em formação na busca dos diplomas, a educação continuada começa na universidade. O constante cuidado de saúde pela equipe de enfermagem aos pacientes é uma tarefa crucial tanto para ofertar qualidade adequada quanto para o acréscimo da profissão (Fernandes et al., 2019).

É importante que se valorizem todas as informações trazidas por profissionais, por isso o acolhimento em grande parte se dá ao ouvir a paciente, dar atenção, falar olhando nos olhos, pois isto demonstra interesse e comprometimento do enfermeiro em relação à história da paciente. Dessa forma, a educação continuada em saúde na enfermagem favorece elementos à população em relação aos fatores de risco, ações de prevenção, encaminhando corretamente as comunidades que apresentam alterações de doenças, além de designar ações que visam diminuir os fatores de risco, diagnosticar e trata-la precocemente (santos-filho, Souza, 2020).

A educação em saúde mostra o entendimento de que o sujeito deve ter o conhecimento com método em atingir um determinado objetivo em seguir sua carreira profissional, tal carreira que incluem meios eficazes de aperfeiçoamento para a realização profissional e ofertar a qualidade integral do atendimento a toda sociedade, sempre avaliando a importância e necessidade do paciente como um todo. A participação operante do enfermeiro no ato de orientar, compartilhar e ajudar o paciente, evidenciando a disponibilidade para o diálogo, além de permitir uma maior percepção e entendimento por parte do paciente. A comunicação é um processo muito importante na assistência de enfermagem e que a qualidade desta assistência depende dos processos de comunicação utilizados.

No âmbito da educação em saúde, a grande maioria dos cursos e residências formam profissionais afastados das indigências de saúde da população e de organização do sistema. Além disso, enquanto que em algumas regiões do país há uma grande oferta de cursos de desenvolvimento na área da Saúde. Para completar, tem-se muitos educadores de serviços que estão desatualizados e precisam aprender novos modos de ensinar (Brasil, 2014).

Dessa maneira, dentro das políticas da educação em saúde, realiza práticas educacionais no âmbito de capacitar os profissionais da saúde sobre um determinado tema, mostrando assim, passagens para a formação de novos profissionais de saúde, aperfeiçoar a toda população cadastrada e usuária no programa das instituições de saúde. Desta maneira, quando 
se revela as práticas educativas em saúde surge a necessidade constante de uma união com toda equipe de profissionais de saúde havendo uma boa relação multiprofissional. A educação em saúde pode ser determinada de distintas atitudes, mas o intuito principal está voltado para a obtenção de informações atuais sobre diversos temas dialogando experiências, habilidades e mudanças comportamentais para o aprimoramento profissional e da assistência integral a toda comunidade (Lopes et al, 2020).

A Estratégia de Saúde da Família (ESF) nada mais é que uma unidade de serviços públicos de saúde voltadas a realizar uma atenção sucessiva nas especialidades de análises básicas com uma equipe multidisciplinar habilitada aumentando as atividades de níveis primários incluindo a rede de promoção, proteção e recuperação (Figueiredo, 2015).

Com isto essa ESF concebe o primeiro acionador da população com o serviço de saúde do município, certificando a referência os âmbitos de contra referência para os diferentes níveis do sistema, desde que esteja coligada na análise do objetivo a necessidade de maior complexidade tecnológica para a licença dos problemas identificados da saúde (Figueiredo, 2015).

A educação dos profissionais atuantes na saúde e na comunidade de maneira em geral são aspectos que requer esforço para se discutir e mostrar atualidades através dos artifícios pedagógicos educativos onde alcancem com eficácia ao público-alvo, desta maneira, para gerar as possíveis atividades da educação por meio de capacitações é preciso ter e criar didática adequada em relação aos aspectos essenciais que encorajem a participação de todos os indivíduos e assim permitam a capacitação integral (Sardinha-Peixoto et al., 2013).

Quando se trata do processo educacional em capacitações de grupos, mostra-se uma possibilidade no meio de transformar as práticas profissionais existentes nas respostas de perguntas e dúvidas, sendo construídas a partir da reflexão das classes dos trabalhadores e a classe que recebe aquela instrução. A educação permanente em saúde acontece no cotidiano das pessoas e organizações (Brasil, 2014).

Assim, a indicação educativa continuada em saúde abordada na capacitação permanente precisa analisar os profissionais como indivíduos de umas artifício em alicerce social de caráter dos saberes práticas, proporcionando de maneira eficaz aspectos de formação para o atendimento assistencial ao longo da vida, pois as informações transmitidas fazem o profissional manter uma realização pessoal para a melhoria da atividade. Assim, a educação continuada em saúde está voltado para educação no exercício de ensino após a graduação, possuindo uma durabilidade concretizada e utiliza artifício de maneira tradicional, analisando os devidos métodos de erradicar os possíveis problemas que impedem um bom 
funcionamento do processo de trabalho e alertar um determinado grupo sobre respectivos conjuntos de temas.

\section{Considerações Finais}

Através do presente estudo foi possível responder a pergunta norteadora que a educação continuada em saúde para enfermeiros, mostra que o mesmo deve manter uma atualização teórica para assim poder aplicar na prática, sempre visando a qualidade de melhor assistência integral ao paciente e toda sua equipe, construção do saber lidarem com processo sentimental do paciente, estes quesitos são discutidos de maneira em geral por 10 artigos da busca no Scientific Eletronic Library.

Com a análise dos periódicos expostos a educação continuada em saúde aos profissionais da enfermagem são aspectos que requer esforço para se discutir e mostrar atualidades através dos artifícios pedagógicos educativos que alcance com eficácia ao público-alvo, desta maneira, para gerar ás possíveis atividades da educação por meio de capacitações é preciso ter e criar didática adequada em relação aos aspectos essenciais que encorajem a participação de todos os indivíduos e assim permitam a capacitação integral.

A educação continuada em saúde indica o artifício de novas metodologias com a parte coletiva de trabalho na saúde. Sendo assim, seus princípios voltados para à pratica de gestão e atendimento a toda população de maneira geral. Os processos da educação em saúde para enfermeiros têm como objetivos a transformação e mudanças das práticas pedagógicas profissionais e da própria disposição do funcionamento do trabalho. A educação continuada em saúde se baseia no processo de ensino-aprendizagem concernente e na probabilidade de modificar-se as práticas de caráter profissional em capacitações rotineiras em melhorias nas condições de vida social

Entender melhor a educação continuada em saúde é um fator primordial em disseminar uma ação mais elaborada a partir do processo de conscientização para um desenvolvimento qualificado de todo o processo de educação em saúde na enfermagem. Para tal, cabem aos enfermeiros e todos os profissionais manterem atualizados sobre os temas relativos sobre a educação continuada em saúde onde o torna mais seguro em lidar com esta prática. No contexto para melhoria da educação em saúde, seria importante todas as instituições que lidam com profissionais de saúde, ofertarem capacitação constante, com distintos temas voltados para a melhoria da qualidade na assistência aos pacientes, sendo também importante 
a implantação de núcleos para a educação permanente em saúde para todos profissionais na rede hospitalar e atenção básica.

\section{Referências}

Assunção, M. L. B., Silva, C. T. S., Alves, C. A. M., Espíndola, M. M. M. (2020). Educação Em Saúde: A Atuação Da Enfermagem No Ambiente Escolar. Rev enferm UFPE on line, 14, e243745.

Brasil, Ministério da Saúde. Guia de educação permanente: a singularidade dos lugares e das pessoas renovando práticas de saúde. Brasília, 2014.

Barcellos, R. M. S. (2020). Educação permanente em saúde: práticas desenvolvidas nos municípios do estado de Goiás. Trab. educ. saúde, vol.18 no.2.

Fernandes, M. C. D., Araújo, A. R. A., Frazão, Cecília M. F. Q., Silva, F. B. B. L., Enders, B. C., Lira, A. L. B. C. (2017). Capacidade De Inferencia Diagnóstica De Enfermeiras Especialistas. Cienc. Enferm, 23(1).

Fernandes, J. C., Cordeiro, B. C., Rezende, A. C., Freitas, D. S. (2020). Competências necessárias ao gestor de Unidade de Saúde da Família: um recorte da prática do enfermeiro. Saúde debate 43(6) .

Ferreira, R. E., Tavares, C. M. M. (2020). Análise da produção tecnológica de três programas de mestrado profissional na área da Enfermagem. Rev. Latino-Am. Enfermagem. 28:e3276.

Figueiras, A. C. M., Puccini, R. F., Silva, E. M. K. (2014) Educação continuada em desenvolvimento infantil para profissionais da atenção primária em saúde: estudo prospectivo do tipo antes-e-depois. Sao Paulo Med. J. 132(4).

Lavich, C. R. P. (2017). Ações de educação permanente dos enfermeiros facilitadores de um núcleo de educação em enfermagem. Rev. Gaúcha Enferm. 38(1). 
Leonor, V. D. (2015). As contribuições da educação continuada em disfagia orofaríngea para a assistência de enfermagem pediátrica em um hospital de ensino. Rev. CEFAC 17(5).

Lopes, O. C. A. (2020). Competências dos enfermeiros na estratégia Saúde da Família, Esc. Anna Nery, 24(2).

Menin, G. E., Pettenon, M. K. (2015). Terminalidade da vida infantil: percepções e sentimentos de enfermeiros. Rev. Bioét. 23(3).

Minayo, M. C. S (Org) (2010). Pesquisa Social; Teoria Método e Criatividade. (29a ed.), Petrópolis: Vozes.

Ortega, M. D. C. B., Cecagno, D., Seva Llor, M. A., Siqueira, H. C. H., Montesinos, M. J. L., Soler, L. M. (2015). Formação acadêmica do profissional de enfermagem e sua adequação às atividades de trabalho. Rev. Latino-Am. Enfermagem 23(3).

Seva-Llo, A. M. (2015). Relatório de enfermagem no hospital. Acta paul. enferm. 28(2).

Pedraza, D. F. (2020). Percepção de enfermeiros sobre o cuidado nutricional à criança na Estratégia Saúde da Família, Saúde Debate Rio De Janeiro, 44(124), 141-151.

Precce, M. L., Moraes, J. R. M. M. (2020). Processo Educativo Com Familiares De Crianças Com Necessidades Especiais De Saúde Na Transição Hospital-Casa. Texto Contexto Enferm [Internet]. 29:e20190075.

Sardinha-Peixoto, L., Cuzatis Gonçalves, L., Dutra, C. T., Tavares, C. M. M., Dantas, C. A. C., Antunes, C. E. (2013). Educação Permanente, continuada e em serviço: desvendando seus conceitos. Revista eletrônica trimestral de enfermagem, (29), 324-340.

Santos Filho, S. B., Souza, K. V. (2020). Metodologia para articular processos de formaçãointervenção -avaliação na educação profissional em enfermagem. Ciência \& Saúde Coletiva, 25(1):79-88. 
Silva, M. C. M., Lopes Júnior, L. C., Nascimento, L. C., Lima, R. A. G. (2016). Fadiga em crianças e adolescentes com câncer sob a perspectiva dos profissionais de saúde. Rev. LatinoAm. Enfermagem, 24

\section{Porcentagem de contribuição de cada autor no manuscrito}

Murilo de Jesus Porto 60\%

David Jesus de Almeida 40\% 